\title{
Pure laparoscopic donor right hepatectomy for adult living donor liver transplantation: initial report from Southeast Asia liver transplant center
}

Worakitti Lapisatepun ${ }^{1}$, Warangkana Lapisatepun ${ }^{2}$, Phuriphong Chanthima ${ }^{2}$, Sunhawit Junrungsee ${ }^{1}$, Anon Chotirosniramit ${ }^{1}$, Settapong Boonsri ${ }^{2}$, Kanya Udomsin ${ }^{1}$, Suraphong Lorsomradee ${ }^{2}$, Trichak Sandhu ${ }^{1}$

\footnotetext{
${ }^{1}$ Department of Surgery, Chiang Mai University, Chiang Mai, Thailand

${ }^{2}$ Department of Anesthesiology, Chiang Mai University, Chiang Mai, Thailand
}

Background: Living donor liver transplantation (LDLT) is widely performed especially in the area which deceased liver donor was scanty including Thailand. Currently, minimally invasive donor hepatectomy is becoming more popular. We began our LDLT program and minimally invasive liver surgery simultaneously on 2015 . We started the first case of pure laparoscopic donor right hepatectomy in 2020. The aim of this study is to present an experience on developing pure laparoscopic donor right hepatectomy for adult living donor liver transplantation in small size living donor liver transplantation center.

Methods: We collected all living liver donors who underwent donor right hepatectomy for modified right lobe graft in our institute from January 1, 2015 to June 30, 2020. The baseline characteristics and surgical outcomes of donors and recipients between conventional open donor right hepatectomy (CODRH) and pure laparoscopic donor right hepatectomy (PLDRH) group were compared.

Results: There were 51 cases of liver transplantation in our center during that period. There were 21 cases of CODRH and four cases of PLDRH using modified right lobe graft. There was neither hand assisted nor laparoscopic assisted donor right hepatectomy in our series. There was no conversion in PLDRH group. Baseline characteristic, Perioperative data and laboratory investigations were not significance difference between two groups. Overall complications after donor right hepatectomy were $33.3 \%$ in CODRH group and $25 \%$ in PLDTH group $(P=0.743)$. The major complication and mortality of the recipient were not significant difference between both groups.

Conclusions: In well-established living donor liver transplantation center which have surgeons who experienced in hepato-biliary and minimally invasive liver surgery. PLDRH can be done safely after the surgeon have trained from high volume LDLT center which had well-established minimally invasive donor hepatectomy program. However, PLDRH should be started in donor who had no anatomical variation.

Corresponding author: Worakitti Lapisatepun

E-mail: wlapisatepun@gmail.com

(c) The Korean Society for Transplantation

This is an Open Access article distributed under the terms of the Creative Commons Attribution Non-Commercial License (http://creativecommons.org/licenses/by-nc/4.0/) which permits unrestricted non-commercial use, distribution, and reproduction in any medium, provided the original work is properly cited. 